TH3D-8

\title{
A SIMPLIFIED MPIE ANALYSIS FOR PLANAR CIRCUITS WITH N-FOLD ROTATIONAL SYMMETRY AND LUMPED ELEMENTS
}

\author{
ABSTRACT
}

Yung-Jinn Chen, Yi-Hsin Pang, and Ruey-Beei Wu

Department of Electrical Engineering, Rm. 340

National Taiwan University, Taipei, Taiwan 10617, ROC

By applying the idea of discrete Fourier transform to exploit the structural rotational symmetry, a simplified MPIE full wave analysis is proposed to handle planar circuits with lumped elements. Applications to symmetrical five-port circuit and power combiner demonstrate the numerical efficiency and accuracy of this technique.

\section{INTRODUCTION}

Electromagnetic problems associated with planar structures of $N$-fold rotational symmetry structures are encountered in some microwave applications such as the matched symmetrical five-port circuit [1], the circular resonators, and the radial power $\mathrm{di}$ viders/combiners [2]. In order to extract precisely the scattering parameters of the $\mathrm{N}$ fold rotational symmetry device, the full wave analysis which can take into account the effects due to fringing field, radiation, and surface wave leakage should be resorted to. In this respect, the mixed potential integral equation (MPIE) formulation distinguishes itself in its flexibility for analyzing arbitrarily shaped planar circuits [3]. However, the relatively heavy requirement in memory storage and CPU time may easily exhaust the available complicated structures.

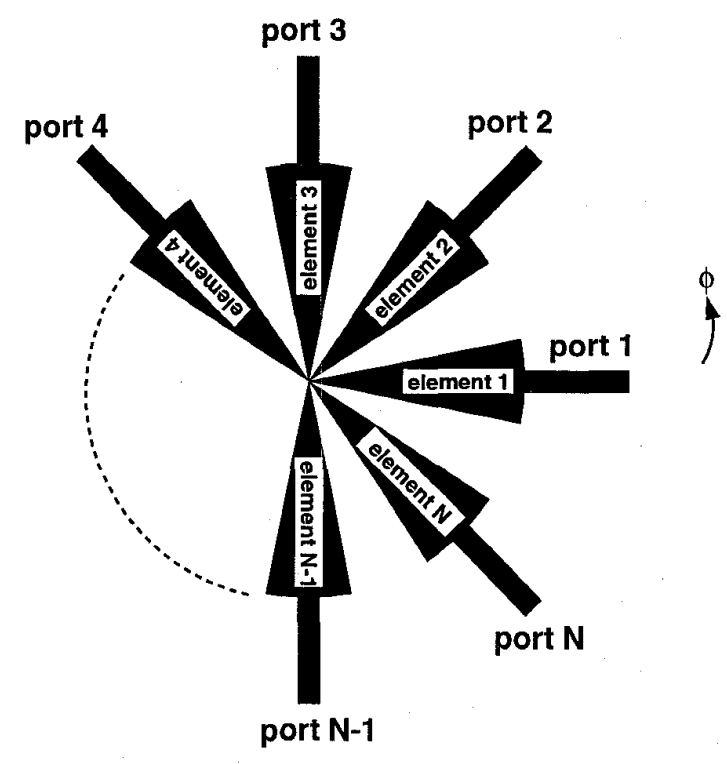

Fig. 1 A typical N-fold rotational symmetry device.

computer resources when it is applied for more TH

\section{HYBRID DFT-MPIE TECHNIQUE}

Fig. 1 shows a typical example for describing how to exploit rotational symmetry in simplifying MPIE solution. Due to the symmetry among the output ports, the single-element excitation can be expressed in terms of a finite discrete spectrum of angular harmonics, by applying the idea of discrete Fourier transform (DFT). For each harmonic, denoted as 
an eigen problem, the current density on the $N$ elements is identical except for a phase shift. Hence, only the current distribution on the first element need be treated as unknown in the MPIE formulation while applying the phase shift relation to the current distribution on all other elements. Consequently, the dimension of the impedance matrix which is resulted by employed the method of moments (MoM) to solve MPIE will be substantially reduced. Given the current distribution $\tilde{\vec{J}}_{k}$ in each eigen problem $(k=0,1, \ldots, N-1)$, the current distribution in any element $n$ of the original problem can be expressed by

$$
\vec{J}_{n}=\sum_{k=0}^{N-1} \tilde{\vec{J}}_{k} e^{j \frac{(n-1) 2 \pi}{N} k}, n=1,2, \ldots, N
$$

It is a well known procedure to solve MPIE for the unknown $\vec{J}$ by applying MoM [3]. Divide the metal into a lot of small cells and choose suitable expansion functions $\vec{B}_{p_{i}}[3]$. By the Galerkin's scheme, a matrix equation will be resulted between voltage vector $[V]$, impedance matrix $[Z]$, and the current coefficients $[I]$. It can be written in the following form

$$
\begin{aligned}
V_{m}= & \sum_{n}\left\{\sum _ { p _ { i } } \sum _ { q _ { j } } T _ { m q _ { j } } \left(j \omega L_{q_{j} p_{i}}+\frac{1}{j \omega C_{q p}}+\right.\right. \\
& \left.\left.R_{q_{j} p_{i}}\right) T_{n p_{i}}\right\} I_{n} \\
= & \sum_{n} Z_{m n} I_{n}
\end{aligned}
$$

where

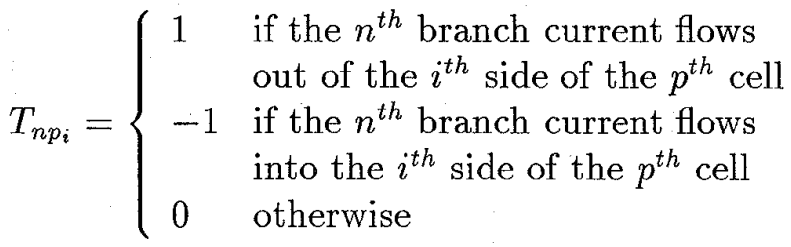

For each eigen problem, say the $k^{\text {th }}$ one, the contributions from the current distribution on all other elements can be added into the first element by using the relationship of the rotational symmetry. Hence, one need only solve the current distribution on the first element, while using (1) to reconstruct those on all the other elements. Consequently, the dimension of the impedance matrix in (2) will be substantially reduced.

For a microstrip structure including isotropic dielectric layers only, the dyadic Green's function $\overline{\bar{G}}_{A}$ in the tangential plane is a scalar function $G_{A}$. Both the potentials $G_{V}$ and $G_{A}$ of an x-directed horizontal electric dipole (HED) are given in the space domain [3]. The matrix elements in (2) should be rewritten to include all contributions coming from other elements. They are in the following form

$$
\begin{gathered}
L_{q_{j} p_{i}}=\sum_{n=0}^{N-1} \int_{\Omega_{q}} \int_{\Omega_{p}} G_{A}\left(x, y \mid x_{n}^{\prime}, y_{n}^{\prime}\right)\left\{B_{q_{j}}^{x}(x, y)\right. \\
{\left[B_{p_{i}}^{x}\left(x^{\prime}, y^{\prime}\right) \cos (n \alpha)-B_{p_{i}}^{y}\left(x^{\prime}, y^{\prime}\right) \sin (n \alpha)\right]+} \\
B_{q_{j}}^{y}(x, y)\left[B_{p_{i}}^{x}\left(x^{\prime}, y^{\prime}\right) \sin (n \alpha)+B_{p_{i}}^{y}\left(x^{\prime}, y^{\prime}\right)\right. \\
\cos (n \alpha)]\} e^{j \alpha k n} d \Omega^{\prime} d \Omega \\
C_{q p}^{-1}=\int_{\Omega_{q}} \int_{\Omega_{p}} \Pi_{q}(x, y)\left[\sum_{n=0}^{N-1} G_{V}\left(x, y \mid x_{n}^{\prime}, y_{n}^{\prime}\right)\right. \\
\left.\quad e^{j \alpha k n}\right] \Pi_{p}\left(x^{\prime}, y^{\prime}\right) d \Omega^{\prime} d \Omega \\
R_{q_{j} p_{i}}=\delta_{p q} Z_{s} \int_{\Omega_{p}} \vec{B}_{q_{j}}(x, y) \cdot \vec{B}_{p_{i}}\left(x^{\prime}, y^{\prime}\right) d \Omega \\
Z_{s}=\sqrt{\frac{j \omega \mu}{\sigma}}
\end{gathered}
$$

where $\alpha=2 \pi / N$, the scalar function $\Pi_{p}=$ $\nabla \cdot \vec{B}_{p_{i}}=1 /\left(\right.$ area of $\left.\Omega_{p}\right), \delta_{p q}$ takes the value of 1 for $p=q$ and 0 for $p \neq q, \mu$ represents the permeability in free space, and $\sigma$ is the conductivity of the conductor. Note that $R_{q_{j} p_{i}}$ keeps unchanged since its value is zero when cell $\mathrm{p}$ is different from cell $\mathrm{q}$. 


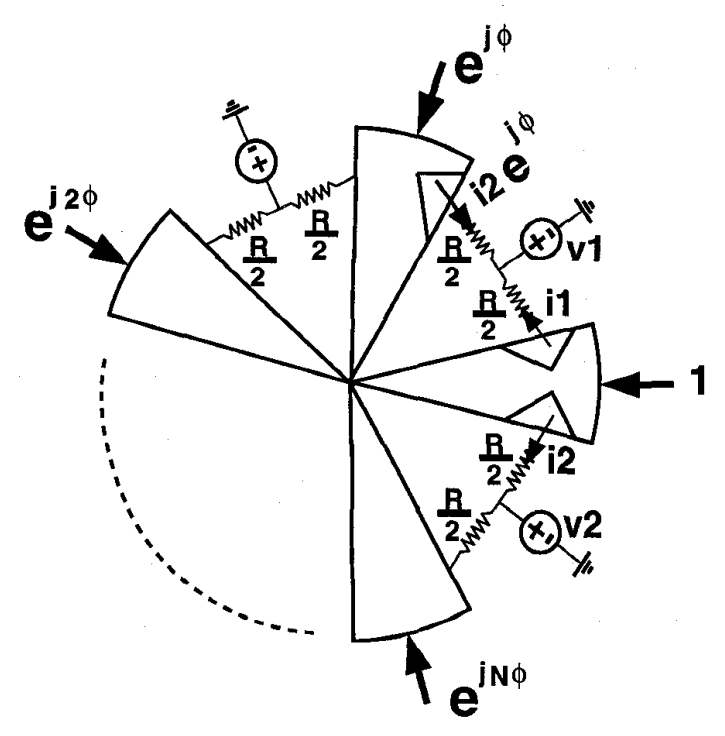

Fig. $2 \mathrm{~N}$-fold rotational symmetry device with isolation resistors.

The scattering parameters can be found from the solved current distribution in each eigen problem. The scattering matrix among all the output ports in the original problem can be constructed from the scattering parameters in the $N$ eigenproblems by applying DFT.

\section{MANAGEMENT OF LUMPED CIR- CUIT ELEMENTS}

In cases that the planar circuits include lumped elements, e.g., isolation resistors in a Wilkinson power combiner, special treatment should be developed to apply the hybrid DFTMPIE technique. Consider the $k^{t h}$ eigen problem shown in Fig. 2, where $R$ denotes the value of isolation resistor and $\phi=\frac{2 \pi k}{N}$, the phase difference between adjacent elements. The virtual voltages $v_{1}, v_{2}$ and the currents $i_{1}, i_{2}$ flowing out of the patch to the resistors satisfy

$$
i_{1}+i_{2} e^{j \phi}=0
$$

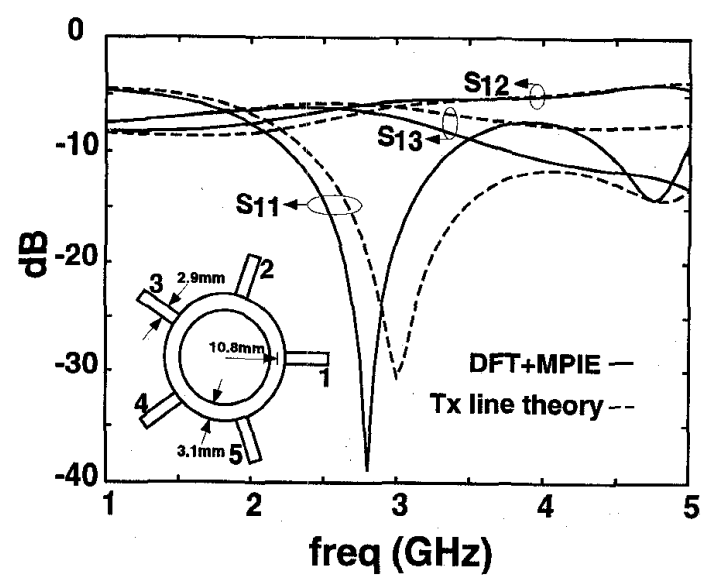

Fig.3 Scattering parameters of the symmetrical fiveport microstrip circuit which is fabricated in the substrate of height $1.5 \mathrm{~mm}$ and dielectric constant 4.33 .

$$
v_{1}-v_{2} e^{j \phi}=0
$$

Treating them as unknowns, the final matrix to be solved will be in the following form [4]

$$
\left[\begin{array}{c}
0 \\
0 \\
0 \\
0 \\
\vdots \\
1 \\
\vdots \\
0
\end{array}\right]=\left[\begin{array}{cccccc}
1 & -e^{j \phi} & 0 & 0 & \cdots & 0 \\
0 & 0 & 1 & e^{j \phi} & \cdots & 0 \\
-1 & 0 & z_{11}+\frac{R}{2} & z_{12} & \cdots & z_{1 n} \\
0 & -1 & z_{21} & z_{22}+\frac{R}{2} & \cdots & z_{2 n} \\
\vdots & \vdots & \vdots & \vdots & \vdots & \vdots \\
\vdots & \vdots & \vdots & \vdots & \cdots & \vdots \\
0 & 0 & z_{n 1} & z_{n 2} & \cdots & z_{n n}
\end{array}\right]\left[\begin{array}{c}
v_{1} \\
v_{2} \\
i_{1} \\
i_{2} \\
\vdots \\
\vdots \\
\vdots \\
i_{n}
\end{array}\right]
$$

where $Z_{i j}$ 's are the aforementioned matrix elements resultant in the MoM solution for MPIE.

It is worthy noting that the approach can be applicable even when the isolation resistors can not be modeled by a simple resistance at higher frequencies. In those cases, the resistance $R$ in (5) should be replaced by the equivalent impedance of the isolation resistors, which can usually be characterized by measurement.

NUMERICAL EXAMPLES 


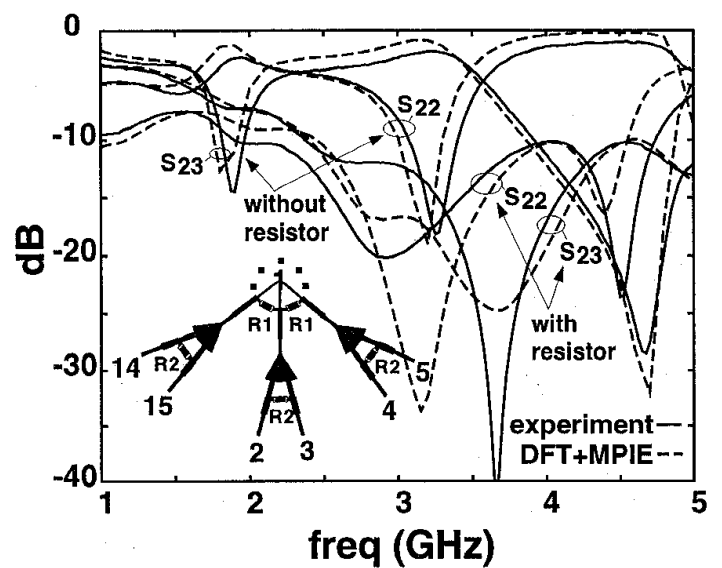

Fig.4 Scattering parameters of a 14-way power combiner

To demonstrate the validity of the hybrid DFT-MPIE technique, a symmetrical five-port circuit is analyzed and the results are compared with those by transmission line theory in Fig. 3. The matched symmetrical five-port circuit is a useful component for a six-port network analyzer [1]. It is found that near the design frequency of $3 \mathrm{GHz}$, the hybrid circuit exhibits desired performance of equal power split into the other four ports and $120^{\circ}$ phase difference between adjacent ports. Although the transmission line theory is helpful in explaining the physical phenomenon, the full wave analysis is necessary to more accurately characterize the circuit and do the fine tune for the final design.

Finally, the technique is applied to analyze a 14-way power combiner proposed in [2, Fig. 5]. Fig. 4 shows the reflection coefficient $S_{22}$ and isolation parameter $S_{23}$ to demonstrate the improvement in isolation by adding the resistors of $R_{1}=30 \Omega$ and $R_{2}=100 \Omega$. The calculated results are found to be in good agreement with the measured data.

\section{CONCLUSIONS}

A numerically efficient technique for com- puting the multiport network scattering matrix of planar structures with $N$-fold rotational symmetry is presented. The technique is based on the solution of MPIE by applying MoM solution in conjunction with both the triangular and rectangular basis functions. The size reduction of the matrix elements is achieved by using DFT. The original problem is divided into $N$ eigen problems such that the number of unknowns is reduced by a factor of $N$ and the overall computational load by $N^{2}$. The matched symmetrical five-port circuit and $N$ way radial power combiner with isolation resistors are used as illustrative examples. $\mathrm{Nu}$ merical results demonstrate the numerical efficiency and accuracy of the present technique.

\section{REFERENCES}

[1] D. I. Kim, K. Araki, and Y. Naito, "Properties of the symmetrical five-port circuit and its broad-band design," IEEE Trans. Microwave Theory Tech., vol. MTT-32, pp. 51-57, Jan. 1984.

[2] Y. J. Chen and R. B. Wu, "A wide band multiport planar power divider design by radially combining matched sectorial components," IEEE MTT-S Dig., pp. 397-400, June 1997.

[3] J. R. Mosig, "Arbitrarily shaped microstrip structures and their analysis with a mixed potential integral equation," IEEE Trans. Microwave Theory Tech., vol. MTT-36, pp. 314-323, Feb. 1988.

[4] D. Koh, R. B. Wu, and T. Itoh, "A hybrid spatial domain analysis of the Wilkinson power divider," Proc. 26th European Microwave Conf., pp. 760-762, Prague, Czech, Sept. 1996. 